\section{Auslöser für Anaphylaxien}

\begin{abstract}
Über den Zeitraum von fünf Jahre konnten die Akten der Notfallambulanz der Kinderklinik des Mount Sinai School of Medicine, New York, ausgewertet werden. Dabei wurden Epidemiologie, Symptome und Verläufe anaphylaktischer Reaktionen genauer unter die Lupe genommen.
\end{abstract}

- $\mathrm{s}$ wurden insgesamt 192 Kinder mit 213 Anaphylaxien behandelt (21 multiple Reaktionen) mit den folgenden Symptomen: v. a. Exantheme/Urtikaria (62\%), Dyspnoe (49\%), Laryngospasmus (42\%), Giemen (26\%), Quincke-Ödem (22\%), Erbrechen (20\%) und Thoraxschmerzen (8\%). Jungen und Mädchen waren gleich häufig betroffen, das mittlere Alter betrug acht Jahre (Median; Range: 0,4 Monate-18 Jahre).

Als häufigste Ursache konnten Lebensmittel identifiziert werden $(71 \%$ der Fälle), dann Medikamente (insgesamt $9 \%$, v. a. NSAR, Antibiotika, Impf- und Hyposensibilisierungs-Lösungen) und $5 \%$ weitere spezifische Auslöser (v.a. Tiere und Insektenstich). Bei $15 \%$ fand sich keine genaue Ursache.

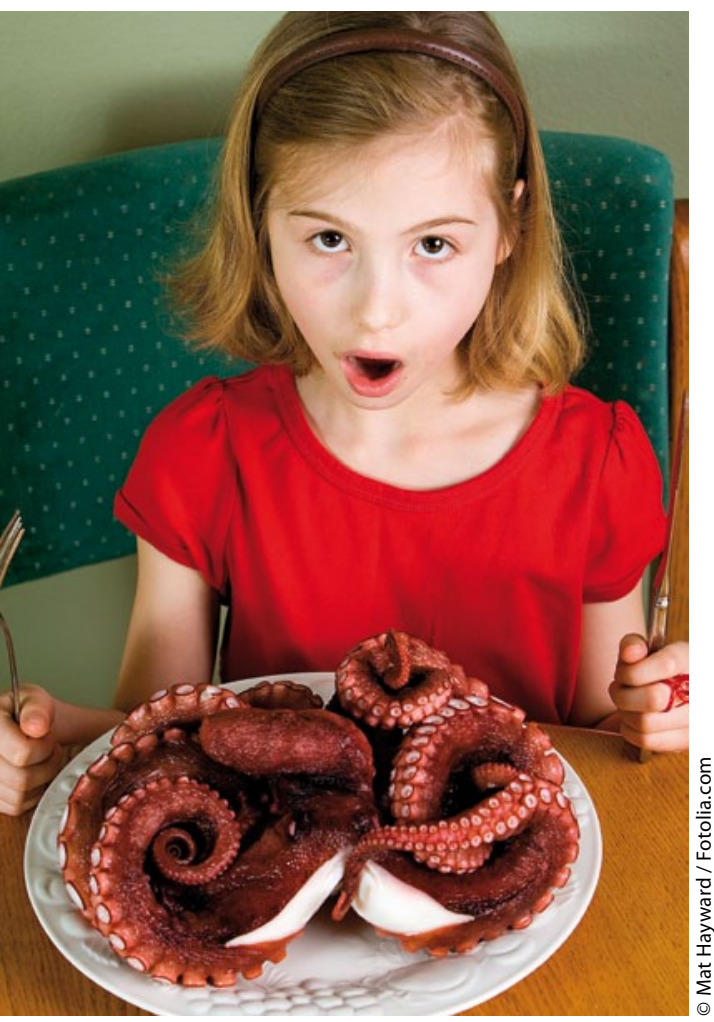

Fisch und Meeresfrüchte sind die häufige Ursachen für eine anaphylaktische Reaktion.
Bei den Nahrungsmittel-Anaphylaxien konnten 85 auf ein einziges spezifisches Lebensmittel zurückgeführt werden, bei 69 waren es „zusammengesetzte“ Lebensmitteln wie z. B. Gebäck. Unter den spezifischen Lebensmitteln führten Fisch und Meeresfrüchte mit $26 \%$, dann Erd- und Baumnüsse mit je $20 \%$. Darüber hinaus gab es Anaphylaxien gegen Gemüse/Obst, Milch, Eier, Weizen, Fleisch und Soja-Produkte. 7 \% der Symptome wurden als schwer und $41 \%$ als mittelschwer klassifiziert; 29 Kinder mussten stationär behandelt werden, neun sogar auf der Intensivstation.

Adrenalin wurde bei 169 (= $79 \%)$ Reaktionen injiziert; bei 58 (=27\%) bereits vor der Ankunft in der Notfallambulanz. In diesen Fällen mussten die Kinder seltener stationär aufgenommen werden $(p=0,05)$. Häufiger als Adrenalin hatten die Kinder Antihistaminika und Kortikosteroide erhalten (79\% bzw. $92 \% / 89 \%)$. Bei 13 (=6\% der Reaktionen) waren zwei Dosen Adrenalin erforderlich, zwei Drittel dieser Patienten blieben stationär, während es sonst nur $12 \%$ waren (Kinder mit einer einzigen Dosis, $\mathrm{p}<0,001)$.

Huang F et al. Anaphylaxis in a New York City pediatric emergency department: triggers, treatments and outcomes. J Allergy Clin Immunol 2012; 129: 162-8.e1-3

Kommentar: Bei Kindern sind Lebensmittel die häufigsten Auslöser von anaphylaktischen Reaktionen; im vorgestellten Untersuchungszeitraum konnte kein Anstieg festgestellt werden. $6 \%$ verlaufen sehr schwer und benötigen eine zweite Dosis Adrenalin. Durch rasche (präklinische) Adrenalin-Injektionen können längere stationäre Behandlungen evtl. vermieden werden. Adrenalin scheint in den USA (zumindest in New York) rascher als hierzulande oder auch in Großbritannien eingesetzt zu werden. Aber auch dort gibt es ein soziales Gefälle: Medicaid-Patienten erhielten seltener Adrenalin.

Dr. Ulrich Mutschler
Anaphylaxie: viele Eltern zu lax Unachtsamkeit von Eltern oder Betreuern spielt bei Nahrungsmittelallergien im Babyalter eine große Rolle. In einer US-amerikanischen Studie mit über 500 Kindern im Alter zwischen drei und 15 Monaten hatten sich $90 \%$ der allergischen Reaktionen ereignet, weil jemand geschludert hatte. Entweder hatten die Eltern nicht gemerkt, was ihr Kind in den Mund steckt, oder sie hatten Etiketten von Nahrungsmittelverpackungen nicht genau gelesen. In etwa der Hälfte aller Fälle waren andere Personen, zum Beispiel die Großeltern, im Spiel.

Vielfach hatten die Eltern selbst einfach vergessen, dass der Sprössling auf ein bestimmtes Lebensmittel "reagiert" obwohl sie im Vorfeld ausführlich aufgeklärt waren. Bei jedem zehnten der insgesamt 1.200 Allergiefälle gaben die Eltern oder Betreuer zu, dem Kind das auslösende Nahrungsmittel bewusst gegeben zu haben. Sie wollten möglicherweise die „verbotene" Speise wieder einführen, ohne jedoch vorher mit einem Arzt gesprochen zu haben. Jede zehnte allergische Reaktion war eine Anaphylaxie, aber nur in jedem dritten Fall wurde Adrenalin verabreicht, vor allem weil die Gefahr nicht erkannt wurde. Dr. Elke Oberhofer

Fleischer DM et al. Pediatrics 2012; 130: e2532

\section{Ohrfeige mit Auswirkung}

Kinder, die mit Schlägen oder Ohrfeigen diszipliniert werden, haben als Erwachsene deutlich häufiger mit psychischen Problemen zu kämpfen. Ungefähr 2-5\% der Achse-I-Störungen bei Erwachsenen, wie u.a. Depression, Manie, Hypomanie, Angststörungen oder soziale Phobien, und 4-7\% der Achse-II-Störungen, wie paranoide sowie schizoide Persönlichkeitsstörungen, Boderline-Erkrankungen oder narzisstische Störungen, gehen laut einer kanadischen Studie auf das Konto wiederholter körperlicher Züchtigung in der Kindheit. Insgesamt 34.653 Teilnehmer wurden befragt, 5,9\% gaben an, derart gemaßregelt worden zu sein. Dabei langten Eltern von Jungen eher mal zu als die von Mädchen. Erstaunlicherweise stieg bei dieser Umfrage mit dem Einkommens- und Bildungsniveau auch der Anteil der Eltern, die ihre Kinder körperlich züchtigten.

Dr. Dagmar Kraus

Afifi TO et al. Pediatrics 2012 Jul 2. [Epub ahead of print] 